Management international

International Management

Gestiòn Internacional

Les priorités du dirigeant face à ses parties prenantes : l'apport

d'une approche centrée sur les attentes

Managing the key issues raised by stakeholders: A

demand-based approach

Las prioridades del dirigente con respecto a sus partes

interesadas: el valor añadido de un modelo orientado hacia las

esperas

Johan BOUGLET et Olivier JOFFRE

Volume 20, numéro 1, automne 2015

URI : https://id.erudit.org/iderudit/1045352ar

DOI : https://doi.org/10.7202/1045352ar

Aller au sommaire du numéro

Éditeur(s)

HEC Montréal

Université Paris Dauphine

ISSN

1206-1697 (imprimé)

1918-9222 (numérique)

Découvrir la revue

Citer cet article

BOUGLET, J. \& JOFFRE, O. (2015). Les priorités du dirigeant face à ses parties prenantes : l'apport d'une approche centrée sur les attentes. Management international / International Management / Gestiòn Internacional, 20(1), 12-25. https://doi.org/10.7202/1045352ar
Résumé de l'article

L'objectif de cet article est de proposer un cadre théorique capable de mieux identifier les priorités du dirigeant. Pour ce faire, nous revenons sur le modèle d'identification des parties prenantes de Mitchell et al. (1997), et montrons qu'une approche par les attentes peut contribuer à le rendre plus opérationnel. Le modèle théorique est ensuite testé à travers l'étude des relations de l'entreprise publique Air France avec ses parties prenantes sur la période 1989-1994. Les résultats montrent que l'approche par les attentes apporte une aide au dirigeant pour anticiper la formation de coalitions.
Tous droits réservés (C) Management international / International Management / Gestión Internacional, 2015
Ce document est protégé par la loi sur le droit d'auteur. L’utilisation des services d’Érudit (y compris la reproduction) est assujettie à sa politique d'utilisation que vous pouvez consulter en ligne.

https://apropos.erudit.org/fr/usagers/politique-dutilisation/ 


\title{
Les priorités du dirigeant face à ses parties prenantes : l'apport d'une approche centrée sur les attentes
}

\author{
Managing the key issues raised by stakeholders: \\ A demand-based approach
}

\section{Las prioridades del dirigente con respecto a sus partes interesadas: el valor añadido de un modelo orientado hacia las esperas}

\author{
JOHAN BOUGLET \\ Institut de Recherche en Gestion,
} Université Paris-Est Créteil Val de Marne

\section{RÉSUMÉ}

Lobjectif de cet article est de proposer un cadre théorique capable de mieux identifier les priorités du dirigeant. Pour ce faire, nous revenons sur le modèle d'identification des parties prenantes de Mitchell et al. (1997), et montrons qu'une approche par les attentes peut contribuer à le rendre plus opérationnel. Le modèle théorique est ensuite testé à travers l'étude des relations de l'entreprise publique Air France avec ses parties prenantes sur la période 1989-1994. Les résultats montrent que l'approche par les attentes apporte une aide au dirigeant pour anticiper la formation de coalitions.

Mots clés : théorie des parties prenantes, attentes, stakeholders. entreprise publique, grève

\author{
OLIVIER JOFFRE \\ Institut de Recherche en Gestion, \\ Université Paris-Est Créteil Val de Marne
}

\section{ABSTRACT}

In this article, we propose a theoretical framework enabling to better identify manager's priority issues. Contributing to the stakeholder identification framework developed by Mitchell et al. (1997), we demonstrate that a demand-based approach may facilitate the operationalization of this very framework. To test this model, we analyze the relations of a public company, namely Air France, with its stakeholders, from 1989 to 1994 . Our findings indicate that the demand-based approach is helpful to the manager in preventing the formation of coalitions.

Keywords: stakeholder theory, demands, stakeholders, public sector, strike

\section{RESUMEN}

El objetivo de este artículo es proponer un marco teórico capaz de identificar mejor las prioridades del dirigente. Para ello, volvemos sobre el modelo de identificación de las partes interesadas de Mitchell y al. (1997), y mostramos que un enfoque por las esperas puede contribuir haciéndolo más operacional. El modelo teórico luego es sometido a un test a través del estudio de las relaciones de la empresa pública Air France con sus partes interesadas sobre el período 1989-1994. Los resultados muestran que el enfoque por las previsiones aporta una ayuda al dirigente para anticipar la formación de coaliciones.

Palabras Claves: teoría de las partes interesadas, esperas, stakeholders. empresa pública, huelga
$\mathrm{L}$ a théorie des parties prenantes appréhende l'entreprise comme une constellation d'intérêts convergents ou divergents (Donaldson et Preston, 1995; Martinet et Reynaud, 2001). Cette approche implique donc la prise en considération des attentes d'une pluralité d'acteurs (stakeholders) dans les décisions de l'entreprise, et non des seuls actionnaires (shareholders), comme le prône le modèle traditionnel de la firme. L'enjeu est de taille, puisqu'il existe un lien entre le management des parties prenantes et rentabilité (Brulhart et Gherra, 2013).

Néanmoins, de nombreux auteurs pointent les limites opérationnelles de cette théorie, notamment quant à la question centrale de l'identification des parties prenantes (Hummels, 1998). Ainsi, Jensen (2002), s'il reconnaît l'importance du concept de partie prenante, souligne que la théorie des parties prenantes (désormais TPP) n'est d'aucune utilité pratique, puisqu'elle ne permet pas au dirigeant d'éta- blir des priorités parmi une pluralité d'objectifs, et ne fournit pas de valeur unique à maximiser. De même, d'autres auteurs (Frooman, 1999; Kochan et Rubinstein, 2000) reprochent à la théorie de ne pas suffisamment considérer les relations entre parties prenantes, et de se limiter à un cadre statique.

En réaction aux critiques formulées, l'objet est ici de proposer un cadre théorique capable de mieux identifier les priorités du dirigeant, en y intégrant les relations entre parties prenantes et la dimension dynamique. Pour cela, la démarche consiste à revenir sur les modèles d'identification des parties prenantes, censés fournir une hiérarchie des priorités du dirigeant, et à utiliser la notion d'attente afin de les compléter. Alors, s'il dispose d'une meilleure vision des enjeux à prendre en compte, le dirigeant sera plus à même de prendre des décisions appropriées. 
La première partie de cette recherche est consacrée à l'examen des modèles d'identification des parties prenantes, et plus particulièrement celui de Mitchell et al. (1997). Nous montrons comment une approche par les attentes peut contribuer à le rendre plus opérationnel. La deuxième partie présente la méthodologie qualitative adoptée pour l'étude du cas Air France sur la période 1989-1994. L'entreprise publique est alors confrontée à une crise sans précédent qui atteint son paroxysme avec la grande grève de 1993. Le PDG, Bernard Attali, doit prendre des décisions cruciales mettant en jeu le personnel de l'entreprise, le gouvernement français, ainsi que la Commission européenne. La troisième partie étudie la dynamique des attentes des parties prenantes à l'aune du modèle théorique proposé. Enfin, dans la dernière partie, nous considérons les implications d'une approche par les attentes dans une double perspective, instrumentale et normative.

\section{Revue de la littérature et cadre conceptuel}

De nombreuses définitions de la notion de partie prenante (désormais PP) existent, au sein d'un continuum allant de l'acceptation la plus large jusqu'à la plus restreinte (Martinet, 1984). La définition qui semble être la plus communément admise est celle donnée par Freeman (1984), selon laquelle une PP est «un individu, ou un groupe d'individus, qui peut affecter ou être affecté par la réalisation des objectifs organisationnels». Il peut dès lors s'agir des actionnaires, fournisseurs, clients, employés, investisseurs financiers, de la communauté, etc. Partant de la TPP, plusieurs modèles d'identification des PP ont été développés (notamment ceux de Savage et al., 1991; Clarkson, 1995; Carroll, 1989; Mitchell et al., 1997). Dans quelle mesure ces modèles permettent-ils d'aider le dirigeant dans l'établissement de ses priorités, en repérant les PP qui méritent le plus d'attention?

\section{Portée et limites OPÉRATIONNELLES DES MODÈLES TRADITIONNELS D'IDENTIFICATION DES PARTIES PRENANTES}

Le modèle d'identification des PP de Mitchell et al. (1997) est considéré comme une référence par une majorité de chercheurs (Neville et al., 2011), car les auteurs reviennent sur les modèles antérieurs, et offrent un modèle de synthèse.

\section{Le modèle de référence de Mitchell et al.}

Mitchell et al. (1997) partent du principe qu'une PP est d'autant plus prioritaire qu'elle présente un nombre élevé de caractéristiques au vu de trois critères : le pouvoir d'influencer les décisions organisationnelles, la légitimité dans les relations avec l'entreprise, et le caractère urgent des droits que les PP peuvent prétendre exercer sur l'entreprise.

Une PP peut être dotée de zéro, un, deux ou trois attributs. En recensant toutes les combinaisons possibles, les auteurs aboutissent à une typologie comportant sept types de PP (un huitième étant constitué des non-parties prenantes), comme le montre la figure 1.

Cette typologie permet d'orienter les priorités du dirigeant vers les PP possédant le plus grand nombre d'attributs

\begin{tabular}{|c|c|c|}
\hline \multicolumn{3}{|c|}{$\begin{array}{c}\text { TABLEAU } 1 \\
\text { Définition des critères d'identification } \\
\text { des parties prenantes }\end{array}$} \\
\hline POUVOIR & LÉGITIMITÉ & $\begin{array}{l}\text { CARACTÈRE } \\
\text { URGENT } \\
\text { DES DROITS }\end{array}$ \\
\hline $\begin{array}{l}\text { Dahl (1957) : «une } \\
\text { relation entre } \\
\text { acteurs sociaux } \\
\text { dans laquelle un } \\
\text { acteur social A peut } \\
\text { obtenir d'un autre } \\
\text { B, de faire quelque } \\
\text { chose qu'il n'aurait } \\
\text { pas fait sinon» }\end{array}$ & $\begin{array}{l}\text { Suchman (1995) : } \\
\text { «une perception } \\
\text { générale que les } \\
\text { actions d'une entité } \\
\text { sont désirables, } \\
\text { convenables ou } \\
\text { appropriées dans } \\
\text { un ensemble } \\
\text { socialement } \\
\text { construit de } \\
\text { normes, valeurs, } \\
\text { croyances et } \\
\text { définitions» }\end{array}$ & $\begin{array}{l}\text { Le caractère urgent } \\
\text { des droits que } \\
\text { les PP peuvent } \\
\text { prétendre exercer } \\
\text { sur l'entreprise } \\
\text { revêt deux aspects : } \\
\text { la sensibilité de } \\
\text { la PP au délai } \\
\text { (Eyestone, 1978), } \\
\text { et le caractère } \\
\text { critique de ces } \\
\text { droits pour la PP } \\
\text { (Hill et Jones, 1992) }\end{array}$ \\
\hline
\end{tabular}

Source : d'après Mitchell et al. (1997).

FIGURE 1

\section{Typologie des parties prenantes d'après Mitchell et al. (1997)}

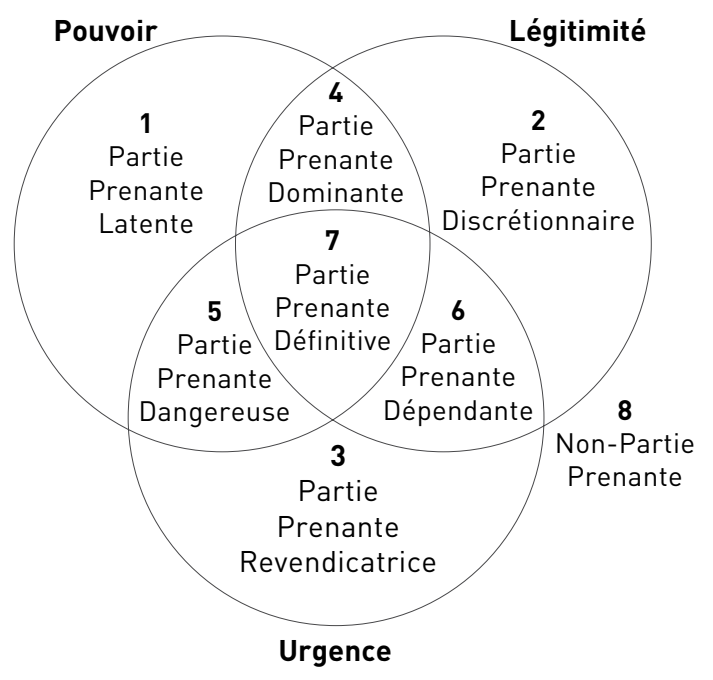

(les PP définitives), ou présentant une combinaison d'attributs nécessitant une attention particulière (les PP dominantes, dangereuses et dépendantes). En cela, elle fournit une aide précieuse au dirigeant.

La nécessité de prendre en compte les relations entre parties prenantes

Frooman (1999) estime que la portée de la plupart des modèles est réduite dans la mesure où ils ne considèrent que des PP isolées. Mercier (2010), dans une analyse historique du concept de PP, estime également que "faire évoluer cette représentation vers la prise en compte d'une plus grande connexion entre les parties prenantes elles-mêmes est fortement souhaitable». 
Cette nécessité se fait d'autant plus sentir que les liens entre PP sont nombreux (Fassin, 2008) et qu'ils ont un impact important sur la structuration de l'environnement. Phillips (2003) montre ainsi qu'une PP peut avoir une influence directe sur les autres.

Selon Cazal (2011), ce manque provient de la vision de la TPP, "obnubilée par l'entreprise et sa centralité, [qui] ne conçoit que des relations dyadiques directes entre l'entreprise et les parties prenantes considérées une par une : aucune forme de collusion, d'alliance entre ces dernières n'est jamais considérée».

Ce constat doit cependant être nuancé. Certains travaux traitent en effet des relations entre PP. Evan et Freeman (1990) définissent l'environnement comme une série de contrats multilatéraux parmi les PP. De même, Rowley (1997) utilise l'analyse des réseaux sociaux pour montrer que les PP pourront, selon leur proximité, contraindre plus ou moins une organisation. D'autres travaux vont plus loin en s'intéressant au phénomène des coalitions. Mitchell et al. (1997) évoquent eux-mêmes leur existence, mais sans les analyser. Dans le cas particulier du secteur public, Nutt et Backoff (1993) recensent ainsi l'existence de coalitions entre PP, et Perrott (1996) montre que certains types de PP sont plus susceptibles que d'autres de former des coalitions contre le dirigeant de la firme. Le dirigeant doit prêter une attention particulière à ce type de relation, eu égard au risque qu'il représente pour la firme. Mais la littérature sur les PP ne fournit pas de méthode analytique pour identifier les coalitions. Freeman (1984) n'aborde lui-même que rapidement le problème lorsqu'il cite la recherche de coalitions comme une étape du processus de formulation de la stratégie par le dirigeant.

Lorsque deux ou plusieurs PP forment une coalition, il semble pourtant possible d'en tenir compte en utilisant le modèle de Mitchell et al. (1997). On peut alors regrouper les attributs respectifs des PP pour signaler leur force jointe. Dans leur modèle, Mitchell et al. (1997) hiérarchisent les PP selon qu'elles présentent un, deux, ou trois des attributs de pouvoir, de légitimité et d'urgence. La question de l'opportunité du regroupement des attributs de deux ou plusieurs PP se pose ici.

En effet, un certain nombre de travaux empiriques tend à montrer que lorsque plusieurs PP forment une coalition, chacune profite des attributs de l'autre (Desreumaux et Romelaer, 2001; Marsch et al., 1988; Dougherty et Hardy, 1996). Mais le modèle de Mitchell et al. (1997) souffre de certaines insuffisances qui empêchent un tel regroupement. Ces dernières sont de différentes natures. Par exemple, une série de critiques renvoie à la portée même du modèle. Les critiques dont il est question ici concernent davantage les concepts mobilisés. En effet, les attributs utilisés sont à l'origine de deux obstacles qui rendent difficile la prise en compte des relations entre les PP et celle de l'aspect dynamique. D'une part, ils sont porteurs d'ambiguïtés. D'autre part, le modèle ne donne pas les moyens de rendre opérationnel l'attribut de pouvoir. Ces deux obstacles sont abordés tour à tour.

Premièrement, les attributs de légitimité et d'urgence présentent des ambiguïtés. Jonker et Foster (2002) vont dans ce sens lorsqu'ils estiment qu'il est nécessaire de considérer plusieurs dimensions afin de pouvoir appréhender les différents attributs. Selon les auteurs, tenir compte uniquement de la PP, comme le font Mitchell et al. (1997), ne suffit pas, et doit être complété à la fois par une analyse de l'enjeu et par celle des processus concernés par la relation. Il faut également prendre en considération le phénomène d'ubiquité de certaines PP : le client d'une banque, par exemple, peut en être aussi le salarié et l'actionnaire (Girard et Sobczak, 2010). Selon la facette considérée, les attributs peuvent alors être différents. Enfin, il est souvent supposé, à tort, que les catégories de parties prenantes ont des préférences homogènes (Wolfe \& Putler, 2002). Mercier (2010) estime à cet égard la nécessité d'appliquer une segmentation plus fine au sein de ces catégories. À la suite de ces auteurs, on peut admettre que les caractéristiques de légitimité et d'urgence ne renvoient pas aux PP en elles-mêmes, mais à leurs attentes. Ainsi, deux attentes d'une même PP peuvent être plus ou moins légitimes, et plus ou moins urgentes.

Dès lors, regrouper les attributs des PP ne permet aucunement de juger de l'intensité de l'influence de ces dernières, puisque le regroupement varie selon les attentes. Il existe alors un risque à raisonner sur la nature des $\mathrm{PP}$, qui consiste en l'attribution d'une capacité d'influence à une PP, alors qu'elle ne dispose de cette dernière que pour une attente isolée. Suite à cette critique, il s'avère que tous les modèles prenant la PP comme unité d'analyse portent les mêmes ambiguïtés.

Deuxièmement, le modèle de Mitchell et al. (1997) semble insuffisamment opérationnel en cela qu'il ne fixe pas de critères pour évaluer l'attribut de pouvoir. Pourtant, les auteurs insistent sur la nécessité de mesurer les deux autres attributs (ainsi, pour le concept d'urgence, ils fournissent des indicateurs de propriété, de sentiment, de prévision et d'exposition). De même, s'il reconnaissent le caractère subjectif de l'attribut de légitimité, les auteurs fournissent des pistes permettant de l'apprécier (Mitchell et al., 1999)². En revanche, ils ne donnent aucun moyen pour savoir ce qui donne du pouvoir à une $\mathrm{PP}$.

\section{Des modèles statiques}

Lanalyse traditionnelle ne considère pas suffisamment l'aspect dynamique pour l'identification des PP (Beaulieu et Pasquero, 2002; Gond et Mercier, 2005). Selon Kochan et Rubinstein (2000), les relations entre la firme et les PP peuvent évoluer, parfois même très rapidement, faisant ainsi varier l'importance d'une PP. De même, Jawahar et McLaughlin (2001) reprochent au modèle de ne pas accorder d'importance aux stratégies que le dirigeant peut mettre en place vis-à-vis des PP. De manière plus large, Fassin (2008) place la variabilité comme la grande absente du modèle des

1. En effet, la légitimité accordée dépend du point de vue adopté. 
PP (variabilité de la dépendance des PP, de leur importance et des relations des PP entre elles).

Ici aussi, la critique doit être nuancée, puisque certains travaux traitent de l'aspect dynamique dans leur analyse des PP. Girard et Sobczak (2010) proposent ainsi une cartographie dynamique des parties prenantes fondée sur leur engagement à l'égard de l'entreprise et à l'égard des principes et valeurs de la responsabilité globale. Dans le cas des firmes publiques, Perrott (1996) montre qu'il existe des PP détenant un pouvoir non négligeable sur la firme, mais qui, étant peu intéressées par l'activité de celle-ci, sont peu menaçantes. L'auteur estime que ces PP peuvent changer dans le temps et devenir soudain concernées par l'activité de la firme. Le dirigeant doit anticiper cette évolution, puisque les PP deviennent alors menaçantes. De même, Nutt et Backoff (1993) montrent qu'une même PP peut être, tour à tour, opposante et partisane des activités de la firme. Dès lors, ici encore, tout l'enjeu pour le dirigeant consiste à anticiper les changements de statut des différentes PP. L'ensemble de ces travaux montre qu'au regard de l'objectif de la théorie, c'està-dire l'établissement de priorités pour le dirigeant, il est indispensable d'introduire la variable temps dans l'analyse, puisque l'importance d'une même PP peut-être différente à deux moments distincts.

\section{Proposition d'Un CADRE THÉORIQUe CENTRÉ SUR LES ATTENTES}

Mercier (2010) parle de la PP comme d'un concept vague et ambigu. Pour lever cette ambiguïté, les différentes typologies de PP utilisent des notions diverses, souvent hétérogènes : Freeman (1984) distingue ainsi les PP internes et externes, là où Caroll (1989) préfère parler de $\mathrm{PP}$ primaires et secondaires, Bonnafous-Boucher et Pesqueux (2006) de PP contractuelles ou diffuses, Phillips (2003) de PP normatives ou dérivatives. De leur côté, Friedman et Miles (2002) classent les PP en quatre groupes en fonction des relations avec l'entreprise : intérêts compatibles ou non, nécessaires (internes) ou contingentes (externes). Fassin (2008) distingue, quant à lui, trois types de PP : les stakeholders (porteurs d'enjeux centraux), les stakewatchers (groupes de pression), et les stakekeepers (régulateurs). Tous ces modèles sont pourtant soumis aux mêmes critiques concernant le manque de prise en compte des relations entre PP et de la dimension dynamique. La notion d'attente, proposée ici, apporte, en premier lieu, un changement de perspective pouvant être appliqué à chacun de ces modèles, malgré leur hétérogénéité.

En outre, la notion d'attente permet d'éviter les ambiguïtés portées par les attributs. En effet, le modèle de Mitchell et al. (1997) considère la nature des PP comme objet d'analyse. Or, il n'est pas possible d'affirmer clairement qu'une PP présente les attributs de légitimité et d'urgence. En effet, le problème est que différentes attentes d'une même PP peuvent avoir des attributs différents. Mais ce problème disparaît si, au lieu de porter sur les PP, l'analyse porte sur les attentes. Une attente correspond à une revendication particulière sur laquelle une ou plusieurs PP comptent obtenir satisfaction. Ce qui est proposé ici est d'affecter les attributs de légiti- mité et d'urgence aux attentes, et non plus aux acteurs, pour juger de la force d'une influence. Ainsi, une attente pourra être légitime, urgente, et/ou portée par une PP possédant du pouvoir.

Il n'existe, à notre connaissance, que peu de travaux plaçant les attentes au centre de leur raisonnement. Le modèle de Sahed-Granger et Boncori (2014) est à cet égard précieux car il met en place des indicateurs traduisant les attentes des PP, ce qui permet de les hiérarchiser. Dans le cas du secteur public, citons Bouglet (2006) et Nutt et Backoff (1993). Cherchant à résoudre les problèmes liés à l'existence d'attentes antagonistes dans les organisations publiques, Nutt et Backoff (1993) raisonnent ainsi en termes d'enjeux. Ceux-ci, en créant des «tensions» dans l'organisation, permettent d'initier des changements satisfaisant les différentes attentes. Si leur problématique diffère de la nôtre, ils utilisent toutefois la notion d'attente et montrent qu'une même attente peut concerner plusieurs PP. Ils ne précisent cependant pas qu'une même PP peut avoir plusieurs attentes différentes. Or, cette précision est importante dans la mesure où elle est à l'origine des ambiguïés des attributs de légitimité et d'urgence.

\section{Attentes et prise en compte des relations entre parties prenantes}

En raisonnant sur les attentes, la prise en compte des relations est simplifiée : en cas de coalition, les attributs se regroupent par attente. Alors, en renversant la perspective, et en se focalisant sur les attentes, le dirigeant dispose d'un outil lui permettant d'analyser des phénomènes, à l'image des coalitions, qui échappaient jusqu'ici à la représentation traditionnelle, et d'en tenir compte dans l'établissement de ses priorités. La gestion des priorités ne se fait donc plus par $\mathrm{PP}$, mais par attente.

Dans cette perspective «renversée», ce sont toujours les attributs mobilisés par Mitchell et al. (1997) qui sont utilisés. Or, l'analyse de ce modèle a montré que les auteurs ne donnent pas les moyens de rendre l'attribut de pouvoir opérationnel. Tout au plus, ils indiquent, à la suite de Pfeffer (1981), que le pouvoir est lié au contrôle des ressources dont la firme a besoin. Allant plus loin dans ce sens, il est possible d'apprécier l'attribut de pouvoir en menant systématiquement une analyse des ressources qui sont indispensables à la firme pour qu'elle mette en ouvre sa stratégie. Pour cela, Nutt et Backoff (1993) suggèrent d'évaluer chaque ressource selon deux axes : son caractère critique («criticality»), qui indique dans quelle mesure elle est indispensable à la firme, et sa disponibilité ("availability»), qui indique la facilité avec laquelle elle peut être mobilisée. Dès lors, une PP a d'autant plus de pouvoir qu'elle possède des ressources critiques pour la firme, et que ces ressources sont difficiles d'accès (mis à part auprès de la PP concernée). La nature des ressources pouvant doter une PP de l'attribut de pouvoir peut être, par exemple, le contrôle ou l'accès aux ressources financières, aux voix lors des votes, ou encore aux voies de sanction par les autorités de régulation ou autres (Eden et Ackermann, 1998). 
En reprenant la terminologie de Mitchell et al. (1997), les différents types d'attente sont qualifiés en considération des attributs qu'ils présentent.

Ici, les dimensions qui sont croisées pour chaque attente sont alors :

- la légitimité dont disposent une ou plusieurs PP, appartenant à la coalition, pour l'attente considérée,

- le caractère urgent des droits qu'une ou plusieurs PP, appartenant à la coalition, peuvent prétendre exercer sur l'entreprise pour l'attente considérée,

- le pouvoir dont disposent une ou plusieurs PP appartenant à la coalition.

On aboutit à sept types d'attentes. Leur dénomination reprend les termes utilisés par Mitchell et al. (1997) dans leur modèle :
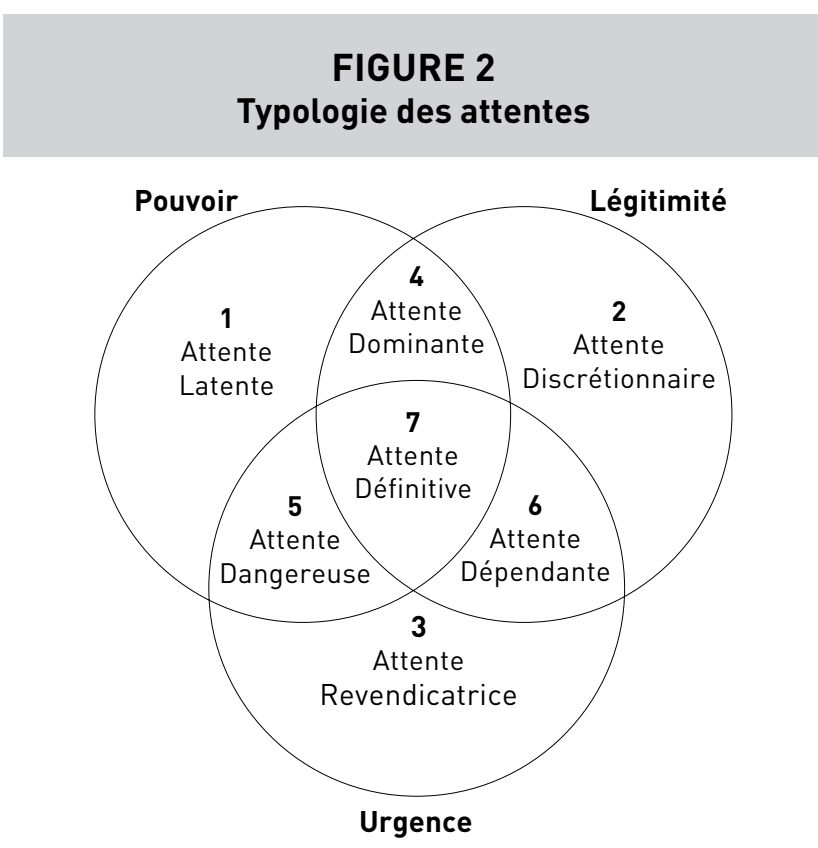

En considérant qu'une coalition est formée d'au moins deux PP, ayant chacune au moins un attribut pour une attente donnée, il s'ensuit que la coalition ainsi formée peut revêtir quatre formes possibles. Certaines attentes résultant d'une coalition possèdent deux attributs (les attentes dominantes, dangereuses et dépendantes), et d'autres en ont trois (les attentes définitives).

Dans une optique instrumentale, cette typologie (figure 2) fournit une aide au dirigeant dans l'établissement de ses priorités. En fonction du type d'attente qu'il aura identifié, le dirigeant pourra apporter une réponse adaptée. Les quatre types d'attente pouvant résulter d'une coalition sont l'attente dominante (4), l'attente dangereuse (5), l'attente dépendante (6) et l'attente définitive (7). Il est possible d'esquisser les enjeux portés par chacun de ces types. Comme les attributs sont différents d'une attente à l'autre, les enjeux sont naturellement d'importances inégales.
Une attente dominante (4) appelle un traitement prioritaire par le dirigeant de la firme considérée. En effet, elle est légitime (on ne peut s'opposer à son fondement), et portée par une coalition ayant du pouvoir. Cela signifie que ne pas en tenir compte peut conduire la firme à être "punie» par les porteurs de l'attente (par exemple, par la voie judiciaire). Rappelons que le caractère "dominant" n'est visible que parce qu'une approche par les attentes est retenue. La vision traditionnelle présenterait les PP séparément, et exigerait un traitement spécifique pour chacune. Grâce à une gestion des attentes, cette relation est directement mise en évidence et incite à apporter un degré de réponse adapté.

Une attente définitive (7) présente les trois attributs. Cela signifie pour le dirigeant qu'elle doit être traitée en priorité et rapidement. Le risque de "punition» est donc encore plus fort que pour une attente dominante si les revendications ne sont pas prises en compte.

Une attente dépendante (6) est détenue par une coalition ne comportant pas de PP possédant un pouvoir sur la firme. Le dirigeant n'a donc a priori pas de raison de la considérer comme prioritaire (l'analyse en univers dynamique montrera qu'il doit néanmoins lui accorder de l'attention).

Enfin, une attente dangereuse (5) mérite toute l'attention du dirigeant. En effet, manquant de légitimité, la concrétisation de cette attente peut s'avérer violente, puisque cette dernière est à la fois urgente et portée par une coalition ayant du pouvoir. Une fois identifiée, elle doit donc faire l'objet d'une attention particulière de la part du dirigeant.

Cette typologie dressée, avec les stratégies de coalition mises en évidence, constitue certainement une aide au dirigeant. Cependant, à l'image de la littérature portant sur la TPP, elle reste statique. Or, les attentes peuvent changer dans le temps, et des contrats informels entre PP peuvent surgir à tout moment. C'est pourquoi l'approche par les attentes doit se doter d'une dimension dynamique.

\section{Une approche dynamique par les attentes}

Les attentes peuvent se modifier de différentes manières : perte ou gain d'attributs, changement d'un attribut en un autre, etc.

Considérant toujours les coalitions comme la menace principale, l'attention se concentre ici sur le cas où les attentes gagnent des attributs. Si l'on considère qu'une attente peut gagner au plus un attribut, il existe douze possibilités de passage d'un type d'attente à un autre. Si l'on considère qu'une attente peut gagner un ou deux attributs, ce nombre passe à quinze. Dans un premier temps, il est donc nécessaire, pour qu'une analyse dynamique des attentes possède un caractère opérationnel, de pouvoir apprécier le risque de formation d'une coalition. Cela permet, dans un deuxième temps, de centrer l'analyse sur les coalitions entre PP qui semblent être les plus probables et les plus dangereuses.

\section{Le risque de formation des coalitions}

Les travaux issus du management public ont mis en évidence l'importance de dégager des priorités dans la gestion des organismes publics (Fredrickson, 1980), notamment en éta- 
blissant une hiérarchie entre les différentes PP au regard de leurs éventuelles coalitions. Il s'agit alors de déterminer les plus risquées.

Premièrement, on peut émettre l'hypothèse que le gain d'un seul attribut est plus probable que celui de deux. Cela signifie que les évolutions les plus susceptibles de se réaliser correspondent au cas où des attentes déjà pourvues d'un attribut se dotent d'un des deux attributs lui manquant grâce à la formation d'une coalition. Perrott (1996) mène une étude empirique confirmant cette hypothèse. Plus précisément, il illustre ces mécanismes à travers l'étude du déploiement du réseau ferroviaire dans la banlieue de Sydney. Il caractérise chaque PP selon deux axes : son pouvoir sur l'organisation et l'intérêt qu'elle a dans les enjeux de celle-ci. Il parvient alors à une matrice comprenant quatre types de PP. L'observation empirique amène ensuite l'auteur à identifier les deux évolutions qui présentent le risque le plus élevé de se réaliser :

- Des PP ayant un pouvoir fort et un intérêt faible forment des coalitions avec des PP ayant un pouvoir faible et un intérêt fort. Ainsi, dans son étude empirique, Perrott (1996) montre que les journaux/médias peuvent relayer les doléances des usagers des nouvelles banlieues qui sont mal desservies;

- Des PP ayant un pouvoir fort et un intérêt faible présentent soudain un intérêt fort.

L'auteur parvient au résultat selon lequel les PP, au travers des coalitions, ne gagnent qu'un seul attribut, et que le gain simultané de deux attributs est moins probable.

Deuxièmement, parmi les cas les plus probables, il est nécessaire de se concentrer sur l'évolution la plus dangereuse : la formation d'une coalition amenant une attente définitive. En, effet, cette dernière ne peut être ignorée du dirigeant puisqu'elle présente les trois attributs existants. Elle peut également prendre plusieurs années à se former. Ainsi, les usagers des nouvelles banlieues de Sydney ont mis sept ans à réunir les attributs afin d'obtenir une situation satisfaisante (Perrott, 1996).

En résumé, l'attention se concentre ici sur le passage d'une attente à deux attributs vers une attente définitive, puisqu'il s'agit de l'évolution la plus risquée pour le dirigeant. Notons que si une attente définitive peut se former à la suite de l'apparition de contrats informels entre plusieurs PP, donnant ainsi naissance à de nouvelles coalitions, elle peut également se former par changement d'une même attente dans le temps (en fonction de l'environnement par exemple).

\section{Les coalitions les plus risquées}

En considérant les différentes catégories d'attente à deux attributs (dominante, dépendante et dangereuse), trois évolutions peuvent mener à une attente définitive, par gain de l'attribut manquant. Quelle que soit la cause de l'évolution, le dirigeant doit prendre en considération l'aspect dynamique pour être en mesure de prévenir le passage d'une catégorie à une autre.
- Passage d'une attente dominante à une attente définitive. Une attente dominante peut se transformer en attente définitive si elle devient urgente.

- Passage d'une attente dépendante à une attente définitive. Alors qu'une attente dépendante ne suppose a priori pas un traitement prioritaire, cela devient pourtant nécessaire si elle intègre l'attribut de pouvoir. Une coalition peut se former non seulement entre les PP présentes au sein d'une même catégorie d'attente, mais aussi par intégration de nouvelles PP, apportant avec elles l'attribut de pouvoir. C'est la raison pour laquelle le dirigeant, une fois l'importance de l'aspect dynamique établi, ne peut pas considérer les attentes dépendantes comme étant moins importantes que les autres : elles peuvent devenir définitives, si des coalitions se forment par contrats informels. En anticipant que l'attente définitive pourra exister et se concrétiser, le dirigeant peut préparer, par exemple, des projets alternatifs.

- Passage d'une attente dangereuse à une attente définitive. Confronté à ce risque, le dirigeant doit faire en sorte que toute attente dangereuse ne devienne pas légitime, ou prévoir une réponse si l'attente définitive se concrétise, en utilisant par exemple des outils de gestion de crise.

\section{Méthodologie}

Nous avons soumis le modèle théorique à l'épreuve des faits, à travers l'étude d'Air France sur la période 1989-1994. Cette démarche empirique est destinée à mettre en évidence la portée opérationnelle d'une analyse fondée sur les attentes.

\section{UNE APPROCHE QUALITATIVE}

Dans le cadre de la TPP, l'environnement de l'entreprise se définit comme un ensemble de relations entre PP (Evan et Freeman, 1990). Or, "quand les frontières entre le phénomène et son contexte ne sont pas clairement évidentes", Yin (1994) préconise de recourir à une méthodologie qualitative. Celle-ci est particulièrement adaptée pour expliquer des phénomènes sociaux complexes (Marshall et Rossman, 1989; Yin, 1990). Notre travail de recherche consiste à qualifier les attentes des PP sur la base d'attributs. Des coalitions peuvent, dès lors, être identifiées. D’un point de vue pratique, la nature des variables étudiées nécessite des indications qualitatives, qui ne peuvent émerger qu'au travers d'une étude de cas approfondie.

\section{Air France face À la grande Grève de 1993}

Incarnant «les ailes de la France», Air France est marquée dès sa création en 1933 par l'influence des pouvoirs publics. Dans ce type d'organisation, le besoin de gérer la complexité engendrée par l'existence d'un ensemble flou de nombreuses parties prenantes se fait particulièrement sentir (Eden et Ackermann, 1988). Face à l'enjeu critique que constitue l'établissement des priorités du dirigeant, l'étude de l'entreprise publique Air France est destinée à valider la portée opérationnelle d'une analyse par les attentes. 
De 1989 à 1994, Air France connaît une crise sans précédent impliquant de nombreuses PP (notamment le personnel, le gouvernement et la Commission européenne) sur fond de libéralisation du transport aérien en Europe ${ }^{2}$. La grève particulièrement violente qui éclate en octobre 1993 (blocage du trafic aérien, occupation des pistes, et affrontement avec les CRS jusque sur les pistes de Roissy-CDG) met l'entreprise au bord de la faillite Elle entraîne le retrait du plan social, ainsi que la démission du PDG Bernard Attali. Ces évènements sans équivalent dans l'histoire de l'entreprise ont suscité une exposition médiatique exceptionnelle. Il est par conséquent aisé de suivre l'évolution des déclarations des parties prenantes d'Air France sur cette période, et d'en inférer la dynamique de leurs attentes.

\section{RECUEIL DES DONNÉES}

A l'instar du travail emblématique de Weick (1993), des données secondaires peuvent être mobilisées pour les études de cas (Koenig, 1996; Chabaud et Germain, 2006; Silverman, 2000). Harris (2001) souligne que l'exploitation de données secondaires présente de nombreux avantages : "réduction des biais déclaratifs, accès à l'information portant sur des événements passés, coût, facilité à mettre en évidence la fiabilité des données, et possibilité de valider des résultats obtenus avec d'autres méthodes de recherche». La collecte des données s'est principalement appuyée sur les articles de presse relatant les événements relatifs à la période étudiée. Nous avons systématiquement parcouru la presse généraliste, ainsi que la presse économique, par le biais de requêtes par motsclés. Cette stratégie de collecte a été poursuivie de manière à atteindre une saturation du terrain, c'est-à-dire "jusqu'à ce que les données recueillies n'apportent plus d'informations nouvelles et que l'information marginale ne remette pas en question les cadres construits " (Drucker-Godard et al., 1999).

Afin d'assurer la triangulation des données, plusieurs sources ont été mobilisées : articles de presse (Le Monde, Les Echos, L'Expansion, The Wall Street Journal, The Sunday Times) et interviews des principales parties prenantes (archives INA et retranscriptions). Le choix des sources s'est justifié par leur pertinence par rapport à l'objectif de recherche (abondance de l'information) et par leur grande maniabilité (l'usage de bases de données numérisées a permis de lancer des requêtes garantissant une exhaustivité des informations sur la période étudiée). Les informations publiques (rapports d'activité) ont également été recherchées. Des ouvrages traitant spécifiquement de l'histoire de l'entreprise (Perri, 1994; Autier et al., 2001; Rossignol, 2009) ont complété le corpus de données. Des travaux de recherche ont également permis de valider ces données (Chatelin, 2001, 2002; Lecat, 2002). Ainsi, «la validité des résultats est notamment renforcée quand plusieurs sources et plusieurs méthodes apportent un "tir groupé» des résultats similaires, concordants ou cohérents.» (Romelaer, 2005).

\section{ANALYSE DES DONNÉES}

L'analyse des données s'est appuyée sur un protocole destiné à accroître la fiabilité des résultats. La base de données constituée a été mise à disposition des deux chercheurs. Ces derniers ont effectué séparément le travail d'identification des PP, de leurs attentes, ainsi que les attributs associés, sur le modèle du tableau 2. A cet égard, il leur était possible de se référer à la partie théorique de l'article, et plus particulièrement aux définitions des attributs (pouvoir, légitimité, caractère urgent des droits) présentées dans le tableau 1. A l'issue de ce traitement, les chercheurs ont confronté leurs conclusions, qui se sont avérées convergentes, les différences constatées relevant principalement des terminologies utilisées pour qualifier les attentes des PP.

\section{Résultats}

Le cas d'Air France est étudié, de manière à mettre en évidence la dynamique des attentes des parties prenantes entre 1989 et 1994, et à identifier des coalitions amenées à peser sur l'établissement des priorités du dirigeant.

\section{IDENTIFICATION DES ATTENTES DES PARTIES PRENANTES}

L'étude d'Air France met en évidence trois périodes distinctes marquées par des évolutions dans les attentes des parties prenantes, que sont le personnel de l'entreprise, représenté par les syndicats, la direction avec à sa tête B. Attali, le gouvernement personnifié par le Premier ministre et le ministre de tutelle, ainsi que la Commission européenne.

1989-1991. En septembre 1989, le Président d'Air France, B. Attali déclare que «la situation financière de la compagnie est aujourd'hui satisfaisante». Pourtant, l'entreprise devient structurellement déficitaire à partir de l'exercice 1990 (717 millions de francs de pertes en 1990, 685 millions en 1991), sous l'effet conjugué de la crise du secteur aérien, et des investissements liés au renouvellement de la flotte. L'Etat a annoncé, depuis 1984, que la compagnie devrait réaliser ses investissements à partir de ses ressources propres. Pour répondre à ces défis, B. Attali présente, en septembre 1991, un plan visant à réduire les dépenses de la compagnie, appelé «Cap 93 ». Cela se traduit par un renforcement du contrôle des effectifs (les recrutements sont bloqués à l'exception du personnel naviguant technique). Les syndicats manifestent contre le plan de rigueur, même s'il n'est question pour l'instant que de non-création d'emplois et de gel des salaires.

1992-septembre 1993. La capacité d'autofinancement de la compagnie devient négative (moins un milliard de francs), alors que le contrat de Plan triennal (91/93) qui lie la compagnie à l'Etat, prévoit des objectifs de rentabilité. Des suppressions d'emplois sont désormais à l'ordre du jour, avec une première vague de 3000 en août 1992. Le plan Cap 93 est décliné en un plan social en octobre 1992 : le Programme de Retour à l'Equilibre (PRE). La question de l'ouverture d'Air

2. Des directives européennes successives $(1987,1990,1993)$ permettent aux compagnies européennes de s'installer librement en Europe ainsi que de déterminer leurs tarifs (Encaoua, 1996). 
France à des capitaux privés, voire de sa privatisation est soumise au Sénat.

En septembre 1993, le nouveau gouvernement, dirigé par Edouard Balladur, durcit la position jusqu'alors adoptée par ses prédécesseurs socialistes, à travers le troisième plan de restructuration en 3 ans. 4000 suppressions d'emplois supplémentaires sont annoncées au mois de septembre. La majorité politique prône désormais la privatisation d'Air France.

octobre 1993 - juillet 1994. Suite au resserrement des conditions financières et de travail demandé par la direction, une crise sociale majeure émerge au sein d'Air France. Le conflit s'étend, amenant les forces de l'ordre à bloquer l'accès aux pistes de l'aéroport de Roissy. Le gouvernement change de position. Le Premier ministre E. Balladur déclare finalement: «toutes les dispositions [sont] prises pour que ce plan ne comporte quasiment aucun licenciement». La crise se solde par le retrait total du plan et la démission de B. Attali remplacé par Christian Blanc en octobre 1993. En novembre 1993, La Commission européenne ouvre une enquête sur Air France pour déterminer si des aides publiques n'ont pas été illégalement accordées à la compagnie aérienne, alors même que la compagnie demande à sa tutelle une importante recapitalisation. Par une décision du 27 juillet 1994, la Commission autorise le gouvernement français à procéder à une recapitalisation, à condition que l'Etat français respecte 16 engagements. Ce sont finalement 20 milliards de francs qui seront apportés par l'Etat au capital de l'entreprise cette même année.

\section{LES AMBIGUïTÉS LIÉES À L'UTILISATION DU MODÈLE De Mitchell et AL.}

L'utilisation du modèle originel de Mitchell et al. (1997) nous amène à présenter les PP de la manière suivante.
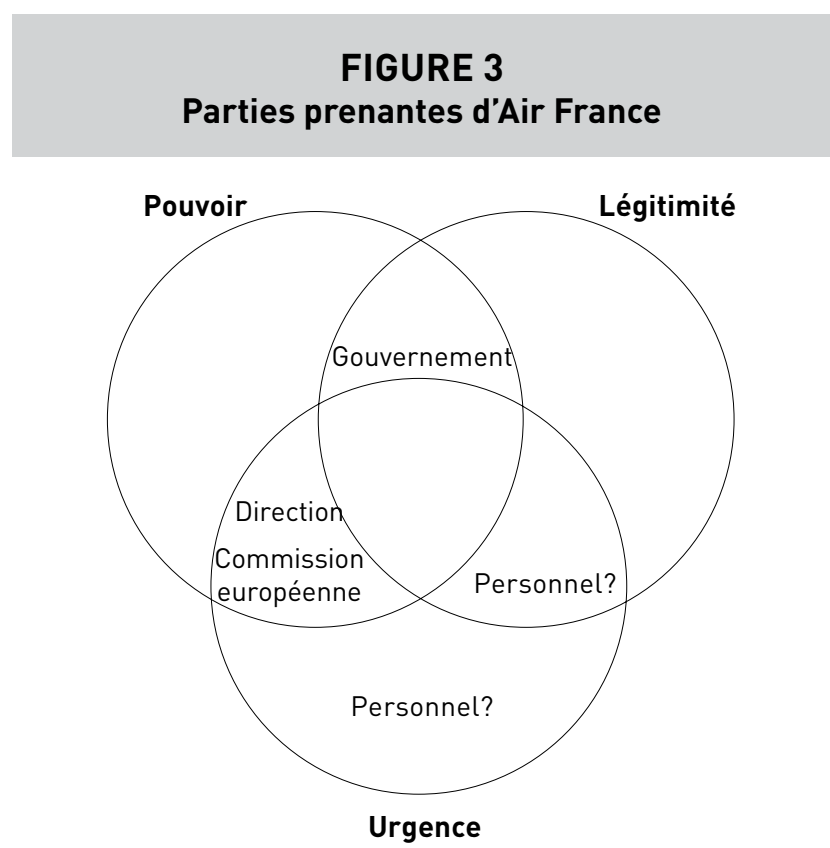

La construction de cette figure pose d'emblée le problème de positionnement de la PP «Personnel». En effet, bien que seulement dotée de l'attribut d'urgence pour l'attente «augmentation des salaires »- ce qui en ferait une PP revendicatrice -, elle présente également l'attribut de légitimité pour l'attente "préservation de l'emploi » - ce qui lui donnerait le statut de PP dépendante -. Cette ambiguité, qui rend l'analyse difficile, sera levée par le modèle centré sur les attentes. Cette figure permet également de voir quaucune PP ne possède les trois attributs conjointement. Or, le jeu des coalitions montrera qu'en unissant des attributs pour certaines attentes, le dirigeant peut identifier des entités "définitives».

Le contexte précédemment décrit permet de recenser les différentes attentes en jeu (tableau 2), et de clarifier ainsi l'ambiguïté venant d'être évoquée. Dans la synthèse proposée, les attentes des PP sont prises isolément. Chacune d'elle est caractérisée par un ou plusieurs des attributs d'urgence $(\mathrm{U})$, de légitimité $(\mathrm{L})$ et de pouvoir $(\mathrm{P})$, ce qui apparaît entre parenthèses.

\section{LA PRISE EN COMPTE DES COALITIONS ENTRE PARTIES PRENANTES}

A la lecture du tableau 2, il apparaît qu'aucune des attentes ne présente les trois attributs.

Par exemple, l'attente "productivité», qui est portée par la direction en 1991, est de ce fait dotée de l'attribut de pouvoir. Elle est également pourvue de l'attribut d'urgence : la direction déclare en effet que "tout retard pris par le groupe dans la modernisation de sa flotte l'entraînerait immanquablement dans une spirale négative de détérioration de sa situation économique et de pertes de parts de marché»" . En revanche, la direction peine à se doter de l'attribut de légitimité, malgré la gravité de la crise qui touche le transport aérien mondial. L'attente de "préservation de l'emploi », portée à la fois par le personnel et le gouvernement sur toute la durée de l'étude, est quant à elle soutenue par l'attribut de légitimité. Perri (1994) rapporte ainsi que «le mouvement à Air France a manifestement réveillé l'opinion [...] 70\% [des français] considèrent la grève comme "une décision légitime».

Une lecture rapide pourrait donner à penser que la situation n'est pas défavorable au dirigeant. Dans un tel cas de figure, ce dernier peut poursuivre ses propres objectifs sans apporter une attention trop importante aux autres attentes, à l'image de B. Attali pendant son mandat.

Toutefois, on remarque que certaines attentes sont communes à plusieurs $\mathrm{PP}$ sur une même période. Il en est ainsi des attentes :

- «productivité» et "préservation de l'emploi» pour la période 1989-1991,

- «éviter la faillite» et "préservation de l'emploi» pour les périodes 1992-1993 et 1993-1994.

Les données recueillies indiquent que seules certaines de ces attentes ont fait l'objet de coalitions ${ }^{4}$ :

3. Les Echos, «Le plan d'Air France pour retrouver l'équilibre en 93», 25/11/1991. 
TABLEAU 2

Les attentes des parties prenantes

PÉRIODES

\begin{tabular}{|l|l|l|l|}
\hline 1989-1991 & $\begin{array}{l}\text { - Augmentation des salaires (U) } \\
\text { - Préservation de l'emploi (L, U) }\end{array}$ & Productivité (P, U) \\
\hline 1992-sept. 1993 & Préservation de l'emploi (L, U) & $\begin{array}{l}\text { Eviter la faillite par mise en } \\
\text { place d'un plan social (P, U) }\end{array}$ & - \\
\hline 0ct.1993-1994 & Préservation de l'emploi (L, U) & $\begin{array}{l}\text { Eviter la faillite par la mise } \\
\text { en place d'un plan social } \\
(P, U)\end{array}$ \\
\hline
\end{tabular}

GOUVERNEMENT

- Productivité $(P, L)$ - Préservation de l'emploi $(P, L)$

Eviter la faillite $(P, L)$

Préservation de l'emploi $(P, L)$

- Eviter la faillite (P, L)

- Préservation de l'emploi (P, L)
COMMISSION EUROPÉENNE

Légende : P (pouvoir), L (légitimité), U (Urgence)

«productivité» pour la période 1989-1991 (coalition entre la direction et le gouvernement); c'est bien l'objet du plan «Cap 93» qui se concrétise par un contrôle des effectifs,

- «éviter la faillite» en 1992-1993 (coalition entre la direction et le gouvernement); la coalition prépondérante de la période précédente renforce sa pression, en annonçant une première vague de 3000 suppressions d'emplois,

- «préservation de l'emploi» en 1993-1994; les plans ne sont plus à l'ordre du jour et leur concepteur, B. Attali, se voit contraint de démissionner.

Une même PP peut donc faire partie de coalitions sur une attente, et rester isolée sur une autre. Ainsi, sur la période 1989-1991, le gouvernement porte deux attentes potentiellement contradictoires (préservation de l'emploi et productivité). Le contexte politique de cette période (les élections législatives n'arrivant qu'en 1993) peut expliquer la prépondérance de l'attente de productivité. Dès lors, l'attente de préservation de l'emploi passe au second plan (les grèves ne trouvent pas d'échos auprès du gouvernement qui ne cherche pas à former une éventuelle coalition).

Conformément au modèle proposé, les attentes peuvent alors être regroupées (elles sont dans ce cas écrites en gras) et réunir leurs attributs (tableau 3).
Les relations mises en évidence concernent des attentes que l'on peut qualifier de "définitives", selon la terminologie de notre typologie. Au sein de la coalition, chaque PP qui les porte voit son pouvoir de négociation renforcé.

La figure 4 permet de visualiser la répartition des attentes pour la première période.

L'attente "productivité» est au centre des préoccupations. Comme B. Attali est lui-même porteur de cette attente, la situation lui est favorable. Le dirigeant n'a donc a priori pas de raison de considérer les deux autres attentes comme prioritaires. Dans les faits, entre 1989 et 1991, les grèves du personnel pour le maintien de l'emploi (caractérisées par les attributs de légitimité et d'urgence) n'ont pas été suivies de mesures allant dans le sens du personnel. Toutefois, la figure 3 montre que la "préservation de l'emploi» est présente à plusieurs niveaux et constitue une menace potentielle.

La période suivante voit évoluer les attentes. Notamment, au regard de la situation financière du groupe qui se dégrade (la capacité d'autofinancement de la compagnie devient négative de un milliard de francs), une nouvelle attente émerge : «Eviter la faillite» (figure 5). Pour le personnel, il ne s'agit plus d'augmenter les salaires mais uniquement de sauvegarder l'emploi.

\begin{tabular}{|c|c|c|c|c|}
\hline \multicolumn{5}{|c|}{$\begin{array}{l}\text { TABLEAU } 3 \\
\text { Mise en évidence des coalitions entre parties prenantes }\end{array}$} \\
\hline PÉRIODES & PERSONNEL & DIRECTION & GOUVERNEMENT & $\begin{array}{l}\text { COMMISSION } \\
\text { EUROPÉENNE }\end{array}$ \\
\hline 1989-1991 & $\begin{array}{l}\text { - Augmentation des salaires (U) } \\
\text { - Préservation de l'emploi (L, U) }\end{array}$ & Productivité $(P, L, U)$ & $\begin{array}{l}\text { - Productivité }(P, L, U) \\
\text { - Préservation de l'emploi }(P, L)\end{array}$ & $\begin{array}{l}\text { Empêcher les aides } \\
\text { illicites de l'Etat }(P, L)\end{array}$ \\
\hline 1992-sept. 1993 & Préservation de l'emploi (L, U) & $\begin{array}{l}\text { Eviter la faillite par } \\
\text { mise en place d'un plan } \\
\text { de productivité }(P, L, U)\end{array}$ & $\begin{array}{l}\text { - Eviter la faillite }(P, L, U) \\
\text { - Préservation de l'emploi }(P, L)\end{array}$ & $\begin{array}{l}\text { Empêcher les aides } \\
\text { illicites de l'Etat (P, L) }\end{array}$ \\
\hline $\begin{array}{l}\text { Oct.1993- } \\
\text { juillet } 1994\end{array}$ & $\begin{array}{l}\text { Préservation de l'emploi } \\
(P, L, U)\end{array}$ & $\begin{array}{l}\text { Eviter la faillite par la } \\
\text { mise en place d'un plan } \\
\text { de productivité }(P, U)\end{array}$ & $\begin{array}{l}\text { - Eviter la faillite }(P, L) \\
\text { - Préservation de l'emploi }(P, L, U)\end{array}$ & $\begin{array}{l}\text { Empêcher les aides } \\
\text { illicites de l'Etat (P, L) }\end{array}$ \\
\hline
\end{tabular}

Légende : P (pouvoir), L (légitimité), U (Urgence)

4. Les autres attentes communes à plusieurs parties prenantes n'ont pas fait l'objet de coalitions. 
FIGURE 4

Les attentes des parties prenantes d'Air France (1989-1991)

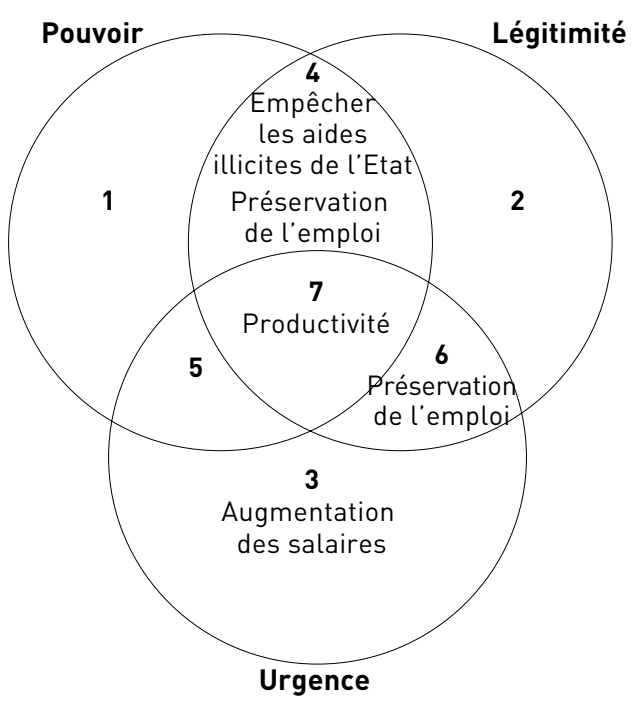

A ce niveau, la situation reste favorable à B. Attali, qui possède l'attente «Eviter la faillite». Toutefois, l'attente de «préservation de l'emploi» est toujours doublement présente. Une approche dynamique des attentes pousse à anticiper son éventuelle évolution. En effet, on s'aperçoit qu'elle porte en elle les trois attributs, même s'ils ne sont pas réunis par l'intermédiaire d'une coalition. Ce risque est matérialisé ici par des flèches convergeant vers les attentes définitives.
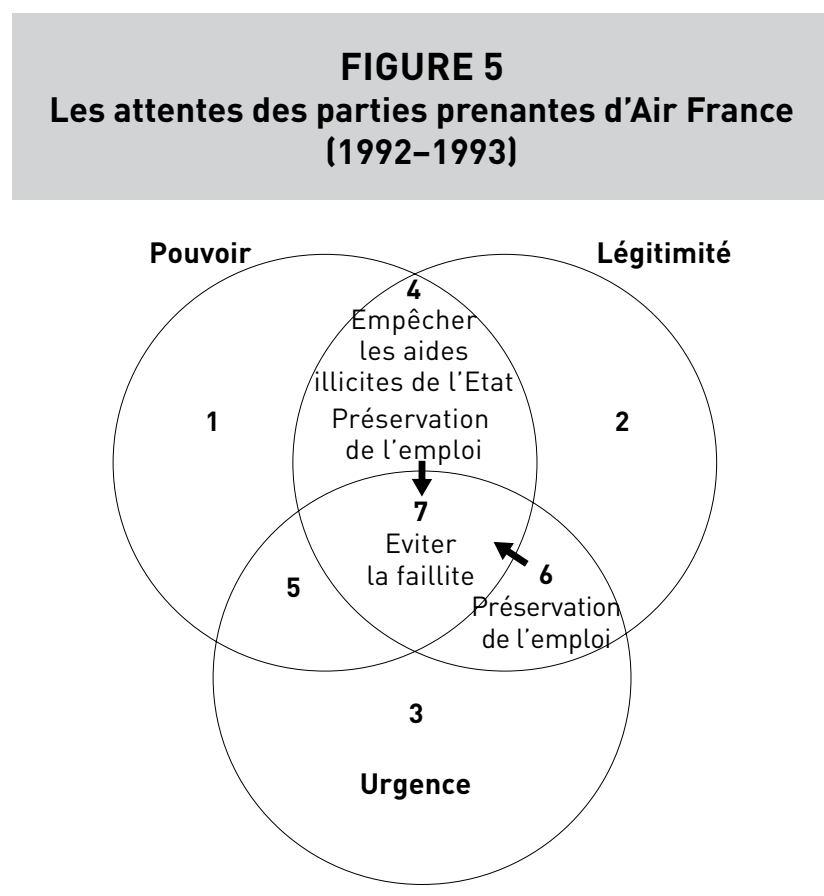

Le risque décrit ci-dessus s'est matérialisé dans le cas d'Air France au cours de la dernière période (1993-1994). En effet, devant le durcissement des conflits sociaux, le gouvernement s'est allié au personnel pour éviter un déferlement médiatique trop fort. Dès lors, l'attente «éviter la faillite» portée par la direction, perd son attribut de légitimité qui était jusque-là porté par le gouvernement. À l'inverse, l'attente «préservation de l'emploi», revendiquée par le personnel, gagne l'attribut de pouvoir grâce à sa coalition avec le gouvernement. Une "volte-face du gouvernement [voulant] manifestement contrecarrer les risques d'extension du conflit dans le secteur public» conduit rapidement à l'éviction de B. Attali, et laisse "place à un "nouveau plan", avec une nouvelle direction. Alors que, durant tout le week-end, le gouvernement campait sur ses positions, refusant [...] toute "remise en cause du plan de retour à l'équilibre", le ministre des Transports, Bernard Bosson a créé la surprise [...] Il a annoncé la mise en chantier d'un nouveau plan social à la faveur d' "une concertation qui, selon l'entourage du ministre, a cruellement manqué au plan Attali». Il y aura bien 4.000 suppressions de poste, mais sans aucun licenciement. "Il faut repartir sur un bon pied, rouvrir le dialogue social. Tout devra être rediscuté $»^{5}$. La convergence d'intérêts a dès lors doté l'attente des trois attributs réunis. A ce moment précis, l'attente devient «définitive» (figure 6).

B. Attali n'avait pas anticipé cette évolution, ou n'avait pas pris les mesures pour la contenir. De plus, le gouvernement
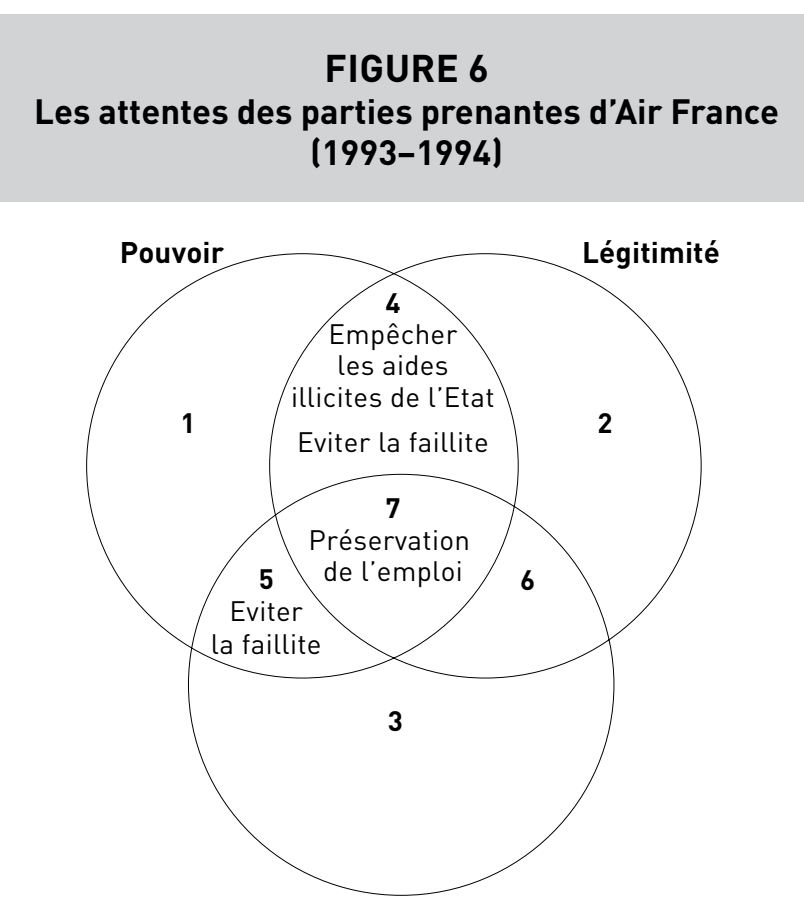

n’a momentanément plus placé l’attente «éviter la faillite» sur le devant. B. Attali a donc perdu un allié, en plus de voir se renforcer ses adversaires. Cette situation s'est soldée par son évincement et la nomination de C. Blanc ${ }^{6}$. 


\section{Discussion}

Le modèle ici développé permet, à notre sens, de participer à l'opérationnalisation de la théorie dans ses différents courants : théorie instrumentale et théorie normative (Donaldson et Preston, 1995) :

La théorie instrumentale des PP (Jones, 1995) stipule que la gestion des PP par le dirigeant peut mener à une meilleure performance de l'entreprise. Dans cette optique, la création de valeur pour chaque PP doit être optimisée afin d'en tirer un avantage compétitif (Mercier, 2006). Weaver et al. (1999) établissent par exemple une relation entre la pression des PP et la formulation des stratégies. Dans cette optique, le dirigeant peut rechercher un intérêt supérieur commun («suprainterest», Bryson et al., 2002) pour éviter la formation d'une coalition contre lui. Cet exercice demande une certaine créativité, puisqu'il s'agit d'imaginer des solutions originales et réalisables. Dans le cas d'Air France, la direction et le gouvernement formaient, dès 1989, une coalition autour de l'attente "productivité». A l'opposé, le personnel restait polarisé sur l'«augmentation des salaires». Il existait des alternatives aux plans de rigueur mis en place par B. Attali : la piste de l'intéressement, qui constituait une revendication des syndicats, aurait permis de réunir les intérêts du gouvernement, de la direction et du personnel. Cette solution est finalement adoptée en 1994 : suite à une vaste consultation interne, C. Blanc présente un plan de redressement prévoyant un échange salaire-actions. Cette mesure est destinée à générer une économie de salaires directs et de charges sociales, de l'ordre de 400 millions de francs par an. Plus généralement, un intérêt supérieur commun peut être trouvé grâce à des politiques de développement durable initiées par les entreprises. Ces dernières peuvent en effet être un outil de création de sens pour les employés et une manière d'éviter le ciblage de l'entreprise par les différentes parties prenantes (Gond, 2006; Joffre et Hamon, 2008).

La théorie normative des PP spécifie quant à elle les obligations morales de l'organisation. Elle considère que les PP doivent être traitées comme des fins. Dans le cas des entreprises publiques, cette approche prend tout son sens, eu égard à leurs missions de poursuite de l'intérêt général. Vernon (1984) montre ainsi que les entreprises publiques ont des objectifs de soutien à l'industrie, de création d'emplois, de sauvegarde de l'intérêt national ou encore de réduction des disparités entre les régions. L'efficacité sociale de ces activités ne peut pas être mesurée par des critères financiers tels que la profitabilité (Pestieau et Tulkens, 1993). Dans ce cadre, l'évaluation de la performance sociale suppose une prise en compte des intérêts de tous les membres de la collectivité (et non de ceux des seuls actionnaires) (François-Marsal, 1973). Le cas Air France est, à cet égard, parlant. Lorsque B. Attali lance le plan «Cap 93» en septembre 1991, c'est clairement la recherche de performance financière qui est visée. Le but affiché est en effet d'améliorer la productivité. Ce faisant, le dirigeant répond à l'une des préoccupations de l'entreprise, mais néglige les autres objectifs attribués aux entreprises publiques. Ses déclarations traduisent cet état d'esprit : «Je trace mon sillon. Je fais mon travail. Au rythme que j'ai choisi, sans me laisser dévier de ma route. On ne construit pas le premier transporteur européen et le troisième mondial avec des états d'âme. L'entreprise subit plusieurs chocs en ce moment, mais c'est la vie naturelle d'une entreprise qui bouge ». En réponse, le Premier ministre alors en place, Edith Cresson, rappelle que les entreprises publiques doivent constituer des «modèles en matière de dialogue social ${ }^{8}$ : des licenciements ne peuvent être annoncés "sans les avoir négociés avec les partenaires sociaux, l'Etat actionnaire et le ministre du Travail ». Cette intervention s'inscrit dans l'approche normative de la TPP : le dirigeant aurait dû tenir compte des revendications des autres PP, au regard des obligations morales d'une entreprise publique.

Enfin, le modèle centré sur les attentes permet d'envisager les interactions entre les courants instrumental et normatif, alors que ces derniers sont souvent considérés séparément. En effet, pour Caillé (2005), la PP de Freeman ne fait que reproduire la figure classique de l'homo economicus animé par une logique maximisatrice standard. Le modèle centré sur les attentes déplace l'analyse, en se focalisant non plus sur des individus mais sur des attentes sociales. Il permet alors d'envisager des solutions facilitant la convergence des intérêts individuels dans le sens de l'intérêt général. Dans le cas d'une entreprise publique comme Air France, les impératifs normatifs en matière de maîtrise des dépenses, de service rendu, et de dialogue social peuvent être atteints, à travers la rencontre des attentes des PP (y compris celles portées par le dirigeant). Ainsi, en 1995, la branche Industries d'Air France a mis en place le Programme d'Innovation Qualité (PIQ) pour encourager le personnel à émettre des propositions destinées à réduire les coûts, ou à améliorer la qualité, les délais, la sécurité, et les conditions de travail. S’appuyant sur un système de récompenses, la démarche a connu un vif succès (1900 idées en 1996, 7700 en 2007). Les économies générées, conséquentes, se sont élevées à 10 millions d'euros sur l'exercice 2006-2007, et témoignent de la capacité du groupe à apprendre de ses échecs. C'est d'ailleurs la recherche de solutions centrées sur les attentes qui a permis au groupe Air France KLM d'éviter un nouvel épisode de grève fin 2013. En effet, le groupe s'est engagé dans un plan de transformation de l'entreprise, «Transform 2015 », avec pour objectifs de retrouver la compétitivité et de repositionner le produit et le service au client au meilleur niveau mondial. Cela implique la suppression de plus de 5000 postes. Un préavis de grève avait été déposé, puis a été levé, car les syndicats se sont dits "très conscients des difficultés que traversent les compagnies aériennes européennes et Air France en particulier "', et ont adhéré à l'attente d'«amélioration de l'efficacité écono-

6. Ce dernier réussira dans les années suivantes à réintégrer les attributs du gouvernement dans l'attente "éviter la faillite», à partir du moment où la Commission européenne autorisera la recapitalisation du groupe par l'Etat.

7. «Bernard Attali : “On ne construit pas le premier transporteur aérien européen avec des états d'âme ”", Les Echos, 12 juin 1991.

8. «Cresson rappelle les « règles du jeu» aux entreprises nationales», Les Echos, 3 octobre 1991. 
mique». De son côté la direction a évité le durcissement du conflit, car l'attente des syndicats « respect des conditions de l'accord» aurait pu se renforcer avec l'intervention du gouvernement dans un contexte politique tendu. La direction, contrairement à ce qu'elle a fait en 1994, a alors suspendu ses projets ${ }^{10}$.

\section{Conclusion}

La recherche de "qui et ce qui compte réellement", chère à Freeman, peut-elle être menée grâce à la théorie des parties prenantes? Les critiques faites au modèle de Mitchell et al. (1997), donnent tout son sens à cette question. En réponse, nous montrons comment une approche par les attentes peut contribuer à doter ce modèle d'une plus grande portée pratique. Les relations entre parties prenantes sont appréhendées de manière dynamique, en appréciant le risque de formation de coalitions entre parties prenantes. La typologie des attentes portées par ces coalitions fournit au dirigeant une aide à la décision, en lui permettant de hiérarchiser ses priorités.

Les relations entre PP sont au cœur du modèle, et leurs évolutions y sont envisagées. Ce dernier point pourrait être encore renforcé. En effet, le modèle ne considère que les attentes des PP déjà identifiées. Pour appréhender l'émergence de nouvelles attentes, il faudrait tenir compte d'attentes simultanées lorsque ces dernières sont en très grand nombre. Appréhender toutes les attentes, y compris les moins prégnantes, permettrait alors de gagner en force prédictive. Une autre limite concerne le nombre de cas étudiés. L'observation d'Air France dans la crise majeure aboutissant à la grande grève d'octobre $1993 \mathrm{a}$, certes permis de conforter l'utilisation du modèle, mais son utilisation devrait être répliquée à d'autres cas afin de renforcer son opérationnalité

Par ailleurs, le renversement de perspective proposé ici a vocation à s'appliquer à d'autres thèmes de recherche. Ainsi, le champ de la gouvernance d'entreprise étudie les forces qui contraignent ou orientent le comportement du dirigeant (Charreaux, 2002). Traditionnellement il s'agit d'étudier les PP. Les mêmes écueils concernant les phénomènes de coalitions et de leur gestion dynamique se posent. Dès lors, étudier les mécanismes sous l'angle des attentes peut constituer une aide méthodologique. Par exemple, le conseil d'administration peut être analysé comme un lieu de rencontre de différentes attentes, dotées d'attributs pouvant ou non s'additionner pour contrôler le dirigeant.

\section{Bibliographie}

AUTIER F., CORCOS G.; TREPO G. (2001), Air France : des années hérö̈ques à la refondation, Vuibert, Collection Management.

BEAULIEU S.; PASQUERO J. (2002), «Reintroducing stakeholder dynamics in stakeholder thinking, A negociated order perspective", in ANDRIOF J., WADDOCK S., HUSTED B. and RAHMAN S., Unfolding Stakeholder thinking. Theory, responsibility and engagement, Sheffield : Greenleaf, p.101-118.

BONNAFOUS-BOUCHER M.; PESQUEUX Y. (2006), Décider avec les parties prenantes, La découverte, coll. Recherches.

BOUGLET J. (2006), «Contribution à la connaissance de la gouvernance d'entreprise : le cas des entreprises nationales", Thèse de Doctorat, Université Paris Dauphine.

BRULHART F.; GHERRA S. (2013), «Management des parties prenantes, pro-activité environnementale et rentabilité : le cas du secteur des produits de grande consommation en France», Finance Contrôle Stratégie, Vol 16, n² 2 , juin 2013.

BRYSON J.M.; CUNNINGHAM G.; LOKKESMOE K.L. (2002), "What to Do When Stakeholders Matter: The Case of Problem Formulation for the African American Men Project of Hennepin County, Minnesota», Public Administration Review, Vol. 65, n² 2, p. 568-584.

CARROLL A.B. (1989), Business and Society : Ethics and Stakeholder Management, South-Western.

CAZAL D. (2011), «RSE et théorie des parties prenantes : les impasses du contrat", Revue de la Régulation, $\mathrm{n}^{\circ} 9$.

CHABAUD D.; GERMAIN O. (2006), «La réutilisation de données qualitatives en sciences de gestion : un second choix?", M@n@gement, Vol. 9, nº 3, p.191-213.

CHARREAUX G. (2002), «Variation sur le thème : A la recherche de nouvelles fondations en finance et gouvernance d'entreprise », Finance Contrôle Stratégie, Vol.5, n³, p.5-68.

CHATELIN C. (2001), " Privatisation et architecture organisationnelle : une contribution à la théorie de la gouvernance à partir d'une approche comparative des formes organisationnelles publiques et privées ", Thèse de Doctorat, Université de Bourgogne, France

CHATELIN C. (2002), «La privatisation d'Air France : un test de la théorie de la gouvernance partenariale», in Sciences de gestion et pratiques managériales, Réseau des IAE, Economica, p. 225-238.

CLARKSON M.E. (1995), "A Stakeholder Framework for Analysing and Evaluating Corporate Social Performance», Academy of Management Review, Vol. 20, p. 92-117.

DAHL R.A. (1957), «The Concept of Power», Behavioral Science, Vol. 2, p. 201-215.

DESREUMAUX A.; ROMELAER P. (2001), «Investissement et organisation", in Images de l'Investissement, Vuibert, Collection Fnege.

DONALDSON T.; PRESTON L.E. (1995), "The Stakeholder Theory of the Corporation : Concepts, Evidence and Implications ", Academy of Management Review, Vol. 20, n 1 , p. 65-91.

9. Les Echos, «Air France : la grève n'aura pas lieu», 18 novembre 2013.

10. Il s'agissait de deux projets portant sur les modalités des journées Absences Enfants Malades et sur l'observation à bord par la Maîtrise. 
DOUGHERTY D.; HARDY C. (1996), «Sustained Product Innovation in Large Mature Organizations : Overcoming Innovation to Organization Problems", Academy of Management Journal, Vol. 39, n 5, p. 1120-1153.

DRUCKER-GODARD, C.; EHLINGER, S.; GRENIER, C. (1999). "Validité et fiabilité de la recherche», in Thiétart et coll., Méthodes de recherches en management, Paris, Dunod, 257-287.

EDEN C.; ACKERMANN F. (1998), Making Strategy : The Journey of Strategic Management, Sage Publications.

ENCAOUA D. (1996), «Ouverture à la concurrence des activités en réseau. Le cas du transport aérien européen ", Revue économique, Vol. 47, nº 6. p.1269-1296.

EVAN W.M.; FREEMAN R.E. (1990), «Corporate Governance : A Stakeholder Interpretation", Journal of Behavioral Economics, Vol. 19, n 4, p.337-359.

EYESTONE R. (1978), From Social Issue to Public Policy, Wiley.

FASSIN Y. (2008), "Imperfections and Shortcomings of the Stakeholder Model's Graphical Representation”, Journal of business ethics, Vol. 80, nº 4 , p. $879-888$.

FRANCOIS-MARSAL F. (1973), Le Dépérissement des Entreprises Publiques, Calmann-Lévy.

FREEMAN R.E. (1984), Strategic Management : A Stakeholder Approach, Pitman.

FRIEDMAN A.L.; MILES S. (2002), «Developing stakeholder theory", Journal of Management Study, Vol. 39, n 1, p. 1-21.

FROOMAN J. (1999), «Stakeholder Influence Strategies», Academy of Management Review, Vol. 24, n 2, p. 191-205.

GIRARD C.; SOBCZAK A. (2010), «Pour une cartographie des parties prenantes fondée sur leur engagement : une application aux sociétaires d'une banque mutualiste française», Management et Avenir, n³3, p.157-174.

GOND J.; MERCIER S. (2005), «Les théories des parties prenantes : une synthèse critique de la littérature», Cahier $d u$ FARGO, $\mathrm{n}^{\circ} 1050502$.

HARRIS H. (2001), "Content Analysis of Secondary Data : A Study of Courage in Managerial Decision Making", Journal of Business Ethics, Vol. 34, n³/4.

HILL C.W.L.; JONES T.M. (1992), "Stakeholder - Agency Theory», Journal of Management Studies, Vol. 29, $\mathrm{n}^{\circ} 2$, p. 131-154.

HUMMELS H. (1998), «Organizing Ethics : A Stakeholder Debate», Journal of Business Ethics, Vol. 17, p. 1403-1419.

JAWAHAR I.M.; MCLAUGHLIN G. L. (2001), "Toward a Descriptive Stakeholder Theory : An Organizational Life Cycle Approach», Academy of Management Review, Vol. 26, $\mathrm{n}^{\circ} 3$, p. 397-414.

JENSEN M. (2002), «Value Maximization, Stakeholder Theory, and the Corporate Objective Function», Business Ethics Quaterly, Vol. 12, n² 2, p. 235-256.

JOFFRE O.; HAMON B. (2008), «Etude d'un modèle de décision dans la coopération agricole : quand la tradition se change en innovation ", Gestion 2000, mai-juin, p. 83-104.

JONES T.M. (1995), "Instrumental Stakeholder Theory : A Synthesis of Ethics and Economics ", Academy of Management Review, Vol. 20, n² 2, p. 404-437.
JONKER J.; FOSTER D. (2002), «Stakeholder Excellence? Framing the Evolution and Complexity of a Stakeholder Perspective of the Firm », Corporate Social Responsibility and Environmental Management, Vol. 9, p. 187-195.

KOCHAN T.A.; RUBINSTEIN (2000), «Toward a Stakeholder Theory of the Firm : The Saturn Partnership ", Organization Science, Vol. 11, $n^{\circ}$ 4, p. 367-386.

LECAT J.-J. (2002), «La propriété et le contrôle des compagnies aériennes. Le cas d'Air France», Revue Internationale de Droit Comparé, Vol.54, n², p.415-440.

MARSCH P., BARWISE P., THOMAS K. and WENSLEY R. (1988), «Managing Strategic Investment Decisions», in A.M. Pettigrew, Competitiveness and the Management Process, Basil Blackwell.

MARSHALL C.; ROSSMAN G.B. (1989), Designing Qualitative Research, Sage Publications.

MARTINET A.C. (1984), Management stratégique : organisation et politique, McGraw-Hill.

MARTINET A.-C.; REYNAUD E. (2001), «Shareholders, stakeholders et stratégie», Revue Française de Gestion, n¹36, novembre-décembre.

MERCIER S. (2006), «La théorie des parties prenantes : une synthèse de la littérature", in Décider avec les parties prenantes, M. BONNAFOUS-BOUCHER et Y. PESQUEUX (coord.), Editions La Découverte, p. 157-172.

MERCIER S. (2010), «Une analyse historique du concept de parties prenantes : Quelles leçons pour l'avenir?», Management \& Avenir, n³3, p.142-156.

MITCHELL R.K., AGLE B.R.; WOOD D.J. (1997), «Toward a Theory of Stakeholder Identification and Salience : Defining the Principle of Who and What Really Counts ", Academy of Management Review, Vol. 22, n 4, p. 853-886.

MITCHELL R.K., AGLE B.R.; SONNENFELD J.A. (1999), «Who Matters to CEOs? An Investigation of Stakeholder Attributes and Salience, Corporate Performance, and CEO Values», Academy of Management Journal, Vol. 42, n 5, p. 507-525.

NEVILLE B.A,; BELL S.J.; WHITWELL G.J. (2011) “Stakeholder Salience Revisited : Refining, Redefining, and Refueling an Underdeveloped Conceptual Tool", Journal of Business Ethics, 102 (3) : pp.357 - 378.

NUTT P.C.; BACKOFF R.W. (1993), «Transforming Public Organizations with Strategic Management and Strategic Leadership ", Journal of Management, Vol. 19, n² 2, p. 299-347.

PESTIEAU P.; TULKENS H. (1993), «Assessing and Explaining the Performance of Public Enterprises», Finanz Archiv, Vol. $50, \mathrm{n}^{\circ} 3$, p. 293-323.

PERRI P. (1994), Sauver Air France, L'Harmattan

PERROTT B.E. (1996), «Managing Strategic Issues in the Public Service», Long Range Planning, Vol. 29, n 3, p. 337-345.

PFEFFER J. (1981), Power in Organizations, Pitman.

PHILLIPS, R. (2003), Stakeholder Theory and Organization Ethics, Berrett-Koehler, San Francisco.

ROMELAER P. (2005), «L'Entretien de recherche», in P. Roussel et F. Wacheux, Management des ressources humaines, De Boeck.

ROSSIGNOL D. (2009), Mutation Economique et Evolution Statutaire, L'Harmattan. 
ROWLEY T.J. (1997), «Moving Beyond Dyadic Ties : a Network Theory of Stakeholder Influence», Academy of Management Review, Vol. 22, n 4, p. 887-910.

SAHED-GRANGER Y.; BONCORI A;-L. (2014), «La traduction des attentes des Parties Prenantes en RSE en indicateurs de la Global Reporting Initiative : vers un mode consultatif de la gestion des parties prenantes", Management et Avenir, Vol.2, $\mathrm{n}^{\circ} 68$.

SAVAGE G.T., NIX T.H., WHITEHEAD C.J. and BLAIR J.D. (1991), "Strategies for Assessing and Managing Organizational Stakeholders», Academy of Management Executive, Vol. 5, p. 61-75.

SILVERMAN, D. (2000), Doing Qualitative Research : A Practical Handbook, Sage.

SUCHMAN M.C. (1995), «Managing Legitimacy : Strategic and Institutional Approaches", Academy of Management Review, Vol. 20, n 3, p. 571-610.

VARLET J. (1997), «La déréglementation du transport aérien et ses conséquences sur les réseaux et sur les aéroports », Annales de Géographie, Vol.106, p. 205-217.

VERNON R. (1984), "Linking Managers with Ministers : Dilemmas of the State-Owned Enterprise », Journal of Policy Analysis and Management, Vol. 4, $\mathrm{n}^{\circ}$ 1, p. 39-55.

WEAVER G.R., TREVINO L.K.; COCHRAN P.L. (1999), «In Press Corporate Ethics Programs as Control Systems : Influences of Executive Commitment and Environment Factors", Academy of Management Journal, Vol. 24, $\mathrm{n}^{\circ} 2$.

WEICK, K. E. (1993), "The Collapse of Sensemaking in Organizations : The Mann Gulch Disaster”, Administrative Science Quarterly, Vol. 38, n 4, p. 628-652.

WILLIAMSON O.E. (1999), «Public and Private Bureaucracy : A Transaction Cost Economic Perspective», Journal of Law, Economics and Organization, Vol. 15, $\mathrm{n}^{\circ}$ 1, p. 306-342.

WOLFE R.; PUTLER D. (2002), "How Tight Are the Ties that Bind Stakeholder Groups", Organization Science, Vol. 13, n ${ }^{\circ}$, p.64-80.

YIN R.K. (1990), Case Study Research, Design and Methods, Sage.

YIN R. K. (1994), Case Study Research, Design and Methods, Sage. 